\title{
Flavonols Inhibit Sortases and Sortase-Mediated Staphylococcus aureus Clumping to Fibrinogen
}

\author{
Sam Sik Kang, ${ }^{a}$ Jae-Gyu KIm, ${ }^{b, c}$ Tae-Hoon LeE, ${ }^{b}$ and Ki-Bong $\mathrm{OH}^{*, b, c}$ \\ ${ }^{a}$ Natural Products Research Institute, College of Pharmacy, Seoul National University; Seoul 110-460, Korea: ${ }^{b}$ School of \\ Agricultural Biotechnology, Seoul National University; Seoul 151-921, Korea: and ${ }^{C}$ Center for Agricultural Biomaterials, \\ Seoul National University; Seoul 151-921, Korea. Received January 5, 2006; accepted March 26, 2006
}

\begin{abstract}
Sortases are a family of Gram-positive transpeptidases responsible for anchoring surface protein virulence factors to the peptidoglycan cell wall layer. In Staphylococcus aureus, deletion of the sortase isoforms results in marked reduction in virulence and infection potential, making it an important antivirulence target. We examined the effects of naturally occurring flavonols on recombinant sortase A (SrtA) and B (SrtB) prepared from $S$. aureus ATCC6538p and found that these compounds inhibited the activity of sortases, without exhibiting antibacterial activities. Among the flavonols tested, morin, myricetin, and quercetin exhibited strong sortase inhibitory activities (SrtA $\left.\mathrm{IC}_{50}: 37.39-52.70 \mu \mathrm{M}, \mathrm{SrtB}_{\mathrm{IC}}: \mathbf{8 . 5 4 - 3 6 . 8 9} \mu \mathrm{M}\right)$. The fibrinogen cell-clumping activity data highlight the potential of flavonols for the treatment of $S$. aureus infections via inhibition of sortase.
\end{abstract}

Key words flavonol; Staphylococcus aureus; sortase; fibrinogen

Bacterial surface proteins constitute a diverse group of molecules with important functions, such as adherence, invasion, signaling, and interaction with the host immune system or the environment. ${ }^{1)}$ In Gram-positive bacteria, many surface proteins are anchored to the cell wall envelope by an enzyme called sortase, which recognizes a conserved carboxylic sorting motif., ${ }^{2,3)}$ Two sortase isoforms, sortase A (SrtA) and sortase B (SrtB), have been identified in Staphylococcus aureus. While the SrtB isoform appears to be important in heme iron acquisition and iron homeostasis, numerous genetic knockout experiments have shown that the SrtA isoform plays a critical role in the pathogenesis of Gram-positive bacteria by modulating the ability of the bacterium to adhere to host tissue via the covalent anchoring of adhesions and other virulence-associated proteins to cell wall peptidoglycan. ${ }^{4-7)}$ SrtA is constitutively expressed and cleaves the amide bond between the threonine and glycine of the LPXTG motif. ${ }^{8-10)}$ Conversely, SrtB recognizes the surface protein substrate bearing the NPQTN motif sorting signal. ${ }^{11)}$ $S$. aureus mutants lacking SrtA fail to display surface proteins and are defective in the establishment of infections without affecting microbial viability. Therefore inhibitors of SrtA might consequently be promising candidates for the treatment and/or prevention of Gram-positive bacterial infections. There have only been a few reports in the literature describing inhibitors of sortase, ${ }^{12-17)}$ due in part to the fact that importance of sortase as a new target has only recently been acknowledged.

Flavonoids represent a large group of plant phenols. These compounds can be classified into flavones, flavonols, flavanones, isoflavones, and anthocyanidins. ${ }^{18)}$ More than 4000 flavonoid compounds have been found. These compounds have many biological and pharmacologic activities including antitumor, antiinflammatory, and antioxidative effects. ${ }^{19}$ Flavonols, the subgroup of flavonoids represented mainly by quercetin and kaempferol, are abundant in fruits and vegetables.

During the course of our search for SrtA inhibitors from medicinal plants, we found that the EtOAc extract from Rhus verniciflua (bark) exhibited strong SrtA-inhibitory activity. ${ }^{20)}$
Bioassay-guided separation of the extract using various chromatographic techniques yielded an active compound. Based on the results of combined spectral analyses and comparison of spectral data with those of known compounds, the active compound was identified as quercetin, because its NMR and EI-MS spectra were in good agreement with those reported previously. ${ }^{21,22)}$ Therefore understanding the structural basis of sortase inhibition by flavonols would provide important clues for the drug design of sortase inhibitors. In this study, we investigated whether natural flavonols inhibit the activity of $S$. aureus sortases and the sortase-mediated $S$. aureus binding to cell-matrix protein fibrinogen.

\section{MATERIALS AND METHODS}

Materials The EtOAc extracts from Rhus verniciflua (bark), which were active in the SrtA inhibition assay, were prepared according to a previously reported procedure. ${ }^{20)}$ Bioassay-guided separation of the extract using various chromatographic techniques yielded an active compound. ${ }^{15)}$ From the results of combined spectral analyses and comparison of the spectral data with those of known compounds (data not shown), the active compound was identified as quercetin, because its ${ }^{1} \mathrm{H}-\mathrm{NMR}\left(500 \mathrm{MHz}\right.$, DMSO- $\left.d_{6}\right)$ spectra showed a pentahydroxyflavone signal with $\delta 6.22(1 \mathrm{H}, \mathrm{d}, J=2.0 \mathrm{~Hz}, \mathrm{H}-$ 6), $6.44(1 \mathrm{H}, \mathrm{d}, J=2.0 \mathrm{~Hz}, \mathrm{H}-8), 6.92(1 \mathrm{H}, \mathrm{d}, J=8.5 \mathrm{~Hz}, \mathrm{H}-$ $\left.5^{\prime}\right), 7.57\left(1 \mathrm{H}, \mathrm{dd}, J=8.5,2.2 \mathrm{~Hz}, \mathrm{H}-6^{\prime}\right)$, and $7.71(1 \mathrm{H}, \mathrm{d}$, $\left.J=2.2 \mathrm{~Hz}, \mathrm{H}-2^{\prime}\right)$; its ${ }^{13} \mathrm{C}-\mathrm{NMR}$ and EI-MS spectra were also in good agreement with those reported previously. ${ }^{21,22)}$ To investigate whether natural flavonols inhibited the activity of $S$. aureus sortases and the sortase-mediated $S$. aureus binding to fibrinogen, we selected eight flavonols structurally related to quercetin from our natural products library: morin, myricetin, quercetin, quercetin-3,3'-dimethyl ether, isorhamnetin, kaempferol, kaempferide, galangin, and galangin3-methyl ether. Fibrinogen (fraction I, type I-S, from bovine plasma) was obtained from Sigma. Fluorescent peptides Dabcyl-QALPETGEE-Edans (for SrtA) and Dabcyl-NPQTNEdans (for SrtB) were synthesized by AnyGen Inc. (Gwangju, Korea). All other reagents used were of the highest grade 
available from commercial sources.

Bacterial Strains S. aureus strain Newman, ${ }^{23)}$ a human clinical isolate, represents the wild-type strain in this study The isogenic sortase knockout mutants SKM12 (srt $A^{-}$), SKM7 $\left(\operatorname{srt} B^{-}\right)$, and SKM14 $\left(\operatorname{srt}^{-} B^{-}\right)$were generated by allelic exchange. ${ }^{6}$ All strains were obtained from Dr. Olaf Schneewind, University of Chicago, Chicago, IL, U.S.A.

Preparation of SrtA and SrtB Recombinant SrtA was prepared according to a previously documented procedure from the overexpressing strain Escherichia coli TOP10 harboring the plasmid pBAD/Thio-TOPO SrtA. ${ }^{24)}$ To acquire $\mathrm{SrtB}$, the $s r t B$ gene was amplified from $S$. aureus ATCC 6538 p genomic DNA by PCR using primers $5 '$-GAATTCGAAGACAAGCAAGAACGC-3' (forward) and 5'-GTCGACTTAACTTACCTTAATTATTTTTGCG-3' (reverse) (GenBank accession no. AP003132), cloned into the pBAD/ThioTOPO vector (Invitrogen), and transformed into $E$. coli TOP10. After arabinose induction of cultures, cells were lysed by lysozyme treatment and sonication, and recombinant SrtB was purified by affinity chromatography on a NiNTA affinity column. ${ }^{24)}$

Sortase Activity Inhibition Assay Sortase inhibitory activity of flavonols was determined by quantifying increased fluorescence intensity upon cleavage of synthetic peptide substrate according to a previously documented procedure. ${ }^{6,17)}$ Briefly, reactions were performed in a volume of $300 \mu \mathrm{l}$ containing Tris- $\mathrm{HCl} 50 \mathrm{~mm}, \mathrm{CaCl}_{2} 5 \mathrm{~mm}, \mathrm{NaCl} 150$ mM, pH 7.5, recombinant $\mathrm{SrtA}_{\Delta 24}$ or $\mathrm{SrtB}_{\Delta 30} 5 \mu \mathrm{M}$, fluorescent peptide substrate Dabcyl-QALPETGEE-Edans (for SrtA) or Dabcyl-NPQTN-Edans (for SrtB) $10 \mu \mathrm{M}$, and prescribed concentrations of the test sample. Each of the test compounds was dissolved in dimethyl sulfoxide (DMSO) and diluted with sterilized distilled water before use (final $1 \%$ DMSO, which was found to have no effect on enzyme activity). Appropriate blanks contained all of the above, with the exception of the test sample. Reactions were carried out for $1 \mathrm{~h}$ at $37^{\circ} \mathrm{C}$ and analyzed fluorometrically (SpectraMAX Gemini XS, Molecular Devices Co., Sunnyvale, CA, U.S.A.) at $350 \mathrm{~nm}$ for excitation and $495 \mathrm{~nm}$ for recordings. $\beta$-Sitosterol-3-O-glucopyranoside, a known SrtA inhibitor, ${ }^{15)}$ was used as a positive control.

Antibacterial Activity Test The effects of flavonols on bacterial growth were determined using the microtiter broth dilution method. ${ }^{25)} S$. aureus strain Newman was grown overnight in brain heart infusion (BHI) broth (Difco), harvested by centrifugation, and then washed twice with sterile distilled water. Each stock solution of test compounds was diluted with $\mathrm{BHI}$ broth to prepare serial dilutions in the range of 300 to $0.1 \mu \mathrm{M}$. One hundred microliters of the broth containing about $10^{5}$ colony forming units (cfu) $/ \mathrm{ml}$ of test bacterium was added to each well of a 96 -well microtiter plate. Culture plates were then incubated for $24 \mathrm{~h}$ at $37^{\circ} \mathrm{C}$, and the absorbance at $600 \mathrm{~nm}\left(\mathrm{OD}_{600}\right)$ was measured using a Multiskan Spectrum spectrophotometer (Thermo Labsystems Inc., Beverly, MA, U.S.A.). The minimum inhibitory concentration (MIC) values were the lowest concentration of test compounds that inhibited cell growth.

S. aureus Clumping Assay The clumping assay was performed as described previously, ${ }^{26}$ ) with the following modifications. The $S$. aureus strain was grown in BHI broth to exponential phase by incubating in a shaking incubator at $37^{\circ} \mathrm{C}$ for $5.5 \mathrm{~h}$. The culture was split into 5 -ml aliquots, and test compound, or control treatment, was added as indicated. After incubation for $2 \mathrm{~h}$ at $37^{\circ} \mathrm{C}$, the cells were harvested by centrifugation at $14000 \mathrm{rpm}$ for $3 \mathrm{~min}$ at $4{ }^{\circ} \mathrm{C}$. Pellets were resuspended in a fibrinogen solution consisting of $1 \mathrm{mg} / \mathrm{ml}$ of fibrinogen in phosphate-buffered salt solution (Dulbecco's PBS $1 \times$, no calcium, no magnesium). The absorbance $\left(\mathrm{OD}_{600}\right)$ was measured for each sample $2 \mathrm{~h}$ after resuspension in the fibrinogen solution. The percent change in absorbance was determined by dividing the absorbance at the time points by that obtained at the initial time point multiplied by 100 .

\section{RESULTS AND DISCUSSION}

Recombinant $\mathrm{SrtA}_{\Delta 24}$ and $\mathrm{SrtB}_{\Delta 30}$ were purified from $E$. coli extracts by metal chelate-affinity chromatography ${ }^{24)}$ and the enzyme activities were determined by quantifying increased fluorescence intensity upon cleavage of synthetic peptide substrate containing either the LPETG or NPQTN motifs. We evaluated the sortase-inhibitory potencies $\left(\mathrm{IC}_{50}\right.$ values) of nine flavonols (Table 1), which we compared with the known SrtA inhibitor $\beta$-sitosterol-3- $O$-glucopyranoside using the assay. $\beta$-Sitosterol-3- $O$-glucopyranoside exhibited potent inhibitory activity against $\mathrm{SrtA}\left(\mathrm{IC}_{50}=31.72 \pm 0.64\right.$ $\mu \mathrm{M}){ }^{15)}$ although little activity against $\mathrm{SrtB}\left(\mathrm{IC}_{50}>300 \mu \mathrm{M}\right)$. Among the flavonols, a hydroxyl group in the B ring ( $4^{\prime}-$ $\mathrm{OH})$, e.g., morin, myricetin, quercetin, quercetin-3,3'-dimethyl ether, isorhamnetin, and kaempferol, conferred more potent inhibitory activity than if this group were lacking, e.g., kaempferide, galangin, and galangin-3-methyl ether. Interestingly, the methylation of the $4^{\prime}$-hydroxyl group in the B ring (kaempferol, SrtA $\mathrm{IC}_{50}=77.94 \pm 6.22 \mu \mathrm{M}$ and $\mathrm{SrtB} \mathrm{IC}_{50}=$ $24.55 \pm 3.08 \mu \mathrm{M}$ ) led to a total loss of activity (kaempferide, $\left.\mathrm{IC}_{50}>300 \mu \mathrm{M}\right)$ against SrtA and SrtB at the highest concentration tested. The importance of hydroxyl groups at $\mathrm{C}-2^{\prime}$ and $\mathrm{C}-4^{\prime}$ in the B ring for high inhibitory capacity of flavonols was found in this study, which also suggests the importance of meta-hydroxyl groups at C-2' and $\mathrm{C}-4^{\prime}$ as in morin $\left(\right.$ SrtA $\mathrm{IC}_{50}=37.39 \pm 3.14 \mu \mathrm{M}, \mathrm{SrtB} \mathrm{IC}_{50}=8.54 \pm 0.30$ $\mu \mathrm{M})$, which was the most potent inhibitor of the compounds tested. Thus morin is more active than myricetin, which bears the $2^{\prime}, 3^{\prime}, 4^{\prime}$-trihydroxy $\mathrm{B}$ ring, and quercetin, which has the ortho-hydroxyl groups at $\mathrm{C}-3^{\prime}$ and $\mathrm{C}-4^{\prime}$ of the $\mathrm{B}$ ring. Moreover, we found that galangin, with a 3-hydroxyl group in the $\mathrm{C}$ ring, was three-fold more active against SrtB than galangin-3-methyl ether. These results suggest that the co-occurrence of the hydroxyl group at $\mathrm{C}-3$ (ring $\mathrm{C}$ ), and meta-hydroxyl groups at $\mathrm{C}-2^{\prime}$ and $\mathrm{C}-4^{\prime}$ (B ring) is essential for flavonol compounds to exhibit strong sortase-inhibitory activity.

Sortase is essential for the functional assembly of surface proteins and for the pathogenesis of $S$. aureus. It has been reported that sortase inhibitors should act as antiinfective agents and disrupt the pathogenesis of bacterial infections without affecting microbial viability. ${ }^{5)}$ Therefore, to rule out effects of test compounds on $S$. aureus cell clumping to fibrinogen due to inhibition of cell growth, the MIC values of flavonols were determined. As shown in Table 1, the flavonols tested in this study exhibited no growth-inhibitory activity against $S$. aureus Newman strain (MIC $>300 \mu \mathrm{M}$ ).

Recent studies ${ }^{6,27)}$ have proposed that $\mathrm{srtB}$ is required for 
Table 1. Inhibitory Effects of Flavonols on the Activity of Sortases (SrtA and SrtB) and the Growth of S. aureus Newman ${ }^{a}$

\begin{tabular}{|c|c|c|c|}
\hline \multirow{2}{*}{ Compound (position of substituent) } & \multicolumn{2}{|c|}{$\mathrm{IC}_{50}(\mu \mathrm{M})$ against } & \multirow{2}{*}{$\operatorname{MIC}(\mu \mathrm{M})$} \\
\hline & SrtA & SrtB & \\
\hline Morin $\left(3,5,7,2^{\prime}, 4^{\prime}-\mathrm{OH}\right)$ & $37.39 \pm 3.14$ & $8.54 \pm 0.30$ & $>300$ \\
\hline Myricetin $\left(3,5,7,3^{\prime}, 4^{\prime}, 5^{\prime}-\mathrm{OH}\right)$ & $44.03 \pm 3.52$ & $36.89 \pm 1.01$ & $>300$ \\
\hline Quercetin $\left(3,5,7,3^{\prime}, 4^{\prime}-\mathrm{OH}\right)$ & $52.70 \pm 1.56$ & $33.28 \pm 2.05$ & $>300$ \\
\hline Quercetin-3,3'-dimethyl ether $\left(5,7,4^{\prime}-\mathrm{OH}, 3,3^{\prime}-\mathrm{OCH}_{3}\right)$ & $53.61 \pm 1.03$ & $60.30 \pm 0.58$ & $>300$ \\
\hline Isorhamnetin $\left(3,5,7,4^{\prime}-\mathrm{OH}, 3^{\prime}-\mathrm{OCH}_{3}\right)$ & $58.86 \pm 0.25$ & $43.35 \pm 0.92$ & $>300$ \\
\hline Kaempferol $\left(3,5,7,4^{\prime}-\mathrm{OH}\right)$ & $77.94 \pm 6.22$ & $24.55 \pm 3.08$ & $>300$ \\
\hline Kaempferide $\left(3,5,7-\mathrm{OH}, 4^{\prime}-\mathrm{OCH}_{3}\right)$ & $>300$ & $>300$ & $>300$ \\
\hline Galangin $(3,5,7-\mathrm{OH})$ & $123.22 \pm 1.93$ & $38.37 \pm 5.89$ & $>300$ \\
\hline Galangin-3-methyl ether $\left(5,7-\mathrm{OH}, 3-\mathrm{OCH}_{3}\right)$ & $117.92 \pm 9.96$ & $113.63 \pm 2.32$ & $>300$ \\
\hline$\beta$-Sitosterol-3-O-glucopyranoside (positive control) & $31.72 \pm 0.64$ & $>300$ & $>300$ \\
\hline
\end{tabular}

a) $n=3$ experiments for each condition.

staphylococcal persistence during infection. The results of those studies indicated that although mutants of $s r t B$ establish infection in renal abscesses and arthritic joints, there was a significant reduction in infective titer over time compared with controls. We observed in our study that morin inhibited SrtB and that the $\mathrm{IC}_{50}$ value against $\mathrm{SrtB}$ was much less than that against SrtA (Table 1). It has been reported that the staphylococcal isd locus encodes anchored surface proteins (IsdA, IsdB, and IsdC) and SrtB, as well as lipoprotein ATPase and membrane permease, which are involved in the transport of heme-iron across the bacterial envelope. ${ }^{6)}$ SrtB recognizes the surface protein substrate IsdC, which carries an NPQTN motif sorting signal. The precise role of IsdC in the physiology of staphylococcal infections is not yet known. While the critical impact of SrtA for the surface display of adhesions and immunomodulatory molecules is well established, the role of SrtB requires further study.

During the sorting signal reaction in $S$. aureus, SrtA is reported to cleave between the threonine and glycine of the LPXTG motif and allow the covalent anchoring of the protein to the bacterial cell wall via a transpeptidation reaction. ${ }^{8,9,28)}$ Proteins such as protein A, clumping factor proteins, and fibronectin-binding proteins are attached to the cell wall by this type of reaction. ${ }^{28)}$ Mutant $S$. aureus strains lacking a functional sortase cannot bind to cell matrix proteins, such as fibrinogen and fibronectin. ${ }^{1,26,29)}$ To begin with, we evaluated the clumping ability of $S$. aureus Newman (wildtype), SKM7 $\left(\operatorname{srt} B^{-}\right), \operatorname{SKM} 12\left(\operatorname{srt} A^{-}\right)$, and SKM14 $\left(\operatorname{srt} A^{-} B^{-}\right)$ to fibrinogen. The change in $\mathrm{OD}_{600}$ was measured for each strain after $2-\mathrm{h}$ incubation at $37^{\circ} \mathrm{C}$ in the fibrinogen solution. The data indicate 75 and $73 \%$ reduction in $\mathrm{OD}_{600}$ from the initial reading by $2 \mathrm{~h}$ for the wild-type and SKM7, respectively (data not shown), indicating significant clumping and precipitation of the bacteria-fibrinogen complex, resulting in this change in absorbance. In contrast, the SKM12 and SKM14 strains showed only 24 and $7 \%$ reduction at $2 \mathrm{~h}$. These results support those previously observed with the wild-type and isogenic sortase knockout mutants. ${ }^{26)}$ They also observed that $s r t A$ plays a critical role in the anchoring of the clumping factor proteins to the cell surface. The absence of the $s r t B$ gene alone does not appear to affect the ability of the strain to bind fibrinogen.

The clumping-inhibitory activity of flavonols was investigated against $S$. aureus Newman (wild-type), and the results are summarized in Fig. 1. The treatment of the wild-type strain with morin, myricetin, quercetin, and kaempferol reduced the capacity of the bacterium to form clumps with fibrinogen in a dose-dependent manner. In the $S$. aureus cellclumping inhibition assay, we observed that morin and quercetin were particularly active. $S$. aureus Newman treated with $100 \mu \mathrm{M}$ of quercetin for $2 \mathrm{~h}$ at $37^{\circ} \mathrm{C}$ exhibited a threefold decrease in the level of fibrinogen cell clumping (increase in $\mathrm{OD}_{600}$ ) after 2 -h incubation at $37^{\circ} \mathrm{C}$ in the fibrinogen solution (Fig. 1). In this experiment, morin exhibited almost the same inhibitory activity against fibrinogen cell clumping as $\beta$-sitosterol-3-O-glucopyranoside. When considering all the data, this means that inhibition of SrtB by morin (Table 1) was not related to the inhibition of fibrinogen cell clumping. The absence of the $s r t B$ gene (SKM7) alone does not appear to affect the ability of the strain to bind fibrinogen, as described above. The clumping ability of the wildtype strain treated with $100 \mu \mathrm{M}$ of quercetin and kaempferide, as representative flavonols, was also compared with that of untreated wild-type strain and SKM12 (Fig. 2). It is important to note that the inhibition of clumping in $S$. aureus treated with quercetin is comparable to the behavior of untreated SKM12.

Flavones and flavonols are usually ingested from vegetables, fruit, tea, and red wine. ${ }^{30,31)}$ The daily intake of flavonoids is estimated to be $23 \mathrm{mg}$ per capita in the Netherlands, $170 \mathrm{mg}$ in the U.S.A., and $70 \mathrm{mg}$ in Japan. ${ }^{32)}$ Flavonoids have antioxidant, antimicrobial, and antiviral activities, and these may contribute to chemopreventive activity. ${ }^{33,34)}$ In addition, recent information suggests that several flavonoids act as inhibitors. ${ }^{35)}$ To our knowledge, this is the first report of the inhibition of $S$. aureus adhesion to fibrinogen via inhibition of sortase by flavonols. It would be important to design specific and powerful inhibitors of this enzyme, which might have 


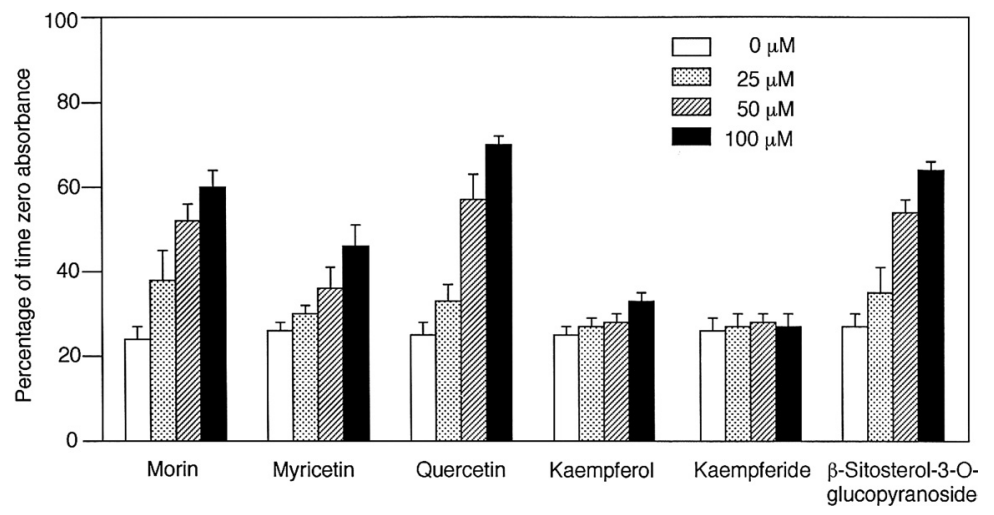

Fig. 1. Inhibitory Effects of Flavonols on the Ability of S. aureus Strain Newman to Clump with Fibrinogen (Mean Value \pm S.D.)

The cultures were treated with indicated concentrations of test compound at $37^{\circ} \mathrm{C}$ for $2 \mathrm{~h}$. Cells were then harvested by centrifugation and resuspended in a fibrinogen solution. Absorbance $\left(\mathrm{OD}_{600}\right)$ measurements were taken for each sample $2 \mathrm{~h}$ after resuspension in the fibrinogen solution. The known SrtA inhibitor $\beta$-sitosterol-3-O-glucopyranoside was used as a positive control.
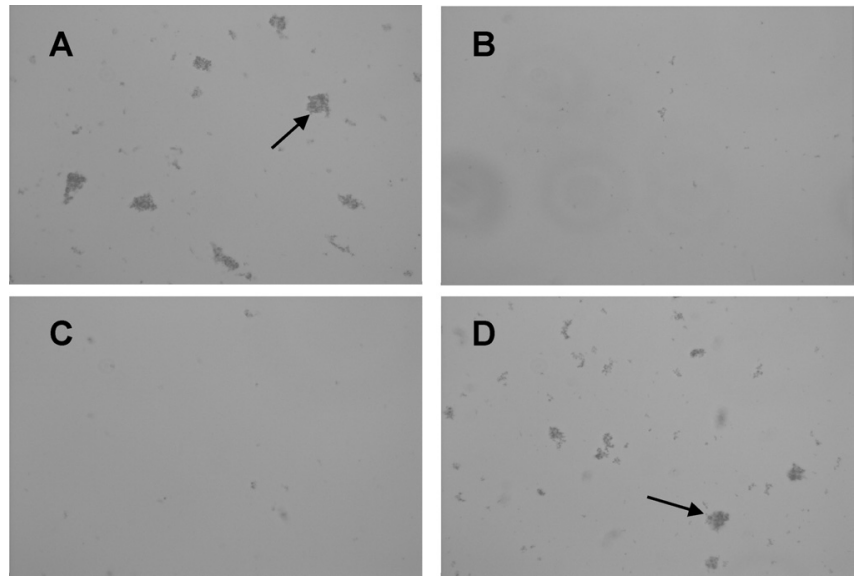

Fig. 2. Comparison of Clumping Activity after Treatment with Representative Flavonols

Pictured are S. aureus: (a) Newman (wild-type), (b) SKM12 srtA-knockout, and (c) wild-type strain treated with quercetin $100 \mu \mathrm{M}$, and (d) wild-type strain treated with kaempferide $100 \mu \mathrm{M}$. Data were recorded $2 \mathrm{~h}$ after resuspension in the fibrinogen solution. Clumps are indicated by arrows.

important applications as a new generation of antibiotics.

In conclusion, naturally occurring flavonols are active against sortases in $S$. aureus and had no antibacterial activity against $S$. aureus growth. The fibrinogen cell-clumping activity data revealed the potential of flavonols for the treatment of $S$. aureus infections via inhibition of sortase activity. The onset and magnitude of inhibition of fibrinogen clumping in $S$. aureus treated with flavonols are comparable to the behavior of untreated sortase knockout mutants. These findings provide the prerequisite for the further study of the structureactivity relationship and in vivo activity on this class of sortase inhibitors.

Acknowledgments This research was supported by a grant (R01-2004-000-10429-0) from the Basic Research Program of the Korea Science and Engineering Foundation. J. G. Kim is the recipient of a fellowship from the Korea Research Foundation Grant funded by the Korean Government (MOEHRD) (KRF-2004-005-F00055). T. H. Lee is the recipient of a fellowship from the Ministry of Education through the Brain Korea 21 Project.

\section{REFERENCES}

1) Alksne L. E., Projan S. J., Curr. Opin. Biotechnol., 11, 625-636 (2000).

2) Mazmanian S. K., Ton-That H., Schneewind O., Mol. Microbiol., 40, 1049-1057 (2001).

3) Navarre W. W., Schneewind O., Microbiol. Mol. Biol. Rev., 63, 174 229 (1999).

4) Mazmanian S. K., Liu G., Ton-That H., Schneewind O., Science, 285 , 760-763 (1999)

5) Mazmanian S. K., Liu G., Lenoy E. R., Schneewind O., Proc. Natl. Acad. Sci. U.S.A., 97, 5510-5515 (2000).

6) Mazmanian S. K., Ton-That H., Su K., Schneewind O., Proc. Natl. Acad. Sci. U.S.A., 99, 2293-2298 (2002).

7) Mazmanian S. K., Skaar E. P., Gaspar A. H., Humayun M., Gornicki P., Jelenska J., Joachmiak A., Missiakas D. M., Schneewind O., Science, 299, 906-909 (2003).

8) Ilagovan U., Ton-That H., Iwahara J., Schneewind O., Clubb R. T., Proc. Natl. Acad. Sci. U.S.A., 98, 6056-6061 (2001)

9) Novick R. P., Trends Microbiol., 8, 148-151 (2000).

10) Ton-That H., Mazmanian S. K., Faull K. F., Schneewind O., J. Biol. Chem., 275, 9876-9881 (2000).

11) Paterson G. K., Mitchell T. J., Trends Microbiol., 12, 89-95 (2004).

12) Connolly K. M., Smith B. T., Pilpa R., Ilangovan U., Jung M. E., Clubb R. T., J. Biol. Chem., 278, 34061-34065 (2003).

13) Frankel B. A., Bentley M., Kruger R. G., McCafferty D. G., J. Am. Chem. Soc., 126, 3404-3405 (2004).

14) Scott C. J., McDowell A., Martin S. L., Lynas J. F., Vandenbroeck K., Walker B., Biochem. J., 366, 953-958 (2002).

15) Kim S. H., Shin D. S., Oh M. N., Chung S. C., Lee J. S., Chang I. M., Oh K. B., Biosci. Biotechnol. Biochem., 67, 2477-2479 (2003).

16) Kim S. H., Shin D. S., Oh M. N., Chung S. C., Lee J. S., Oh K. B., Biosci. Biotechnol. Biochem., 68, 421-424 (2004).

17) Oh K. B., Kim S. H., Lee J., Cho W. J., Lee T., Kim S., J. Med. Chem., 47, 2418-2421 (2004).

18) Beecher G. R., J. Nutr., 133, 3248S-3254S (2003).

19) Ross J. A., Kasum C. M., Annu. Rev. Nutr., 22, 19-34 (2002).

20) Kim S. W., Chang I. L., Oh K. B., Biosci. Biotechnol. Biochem., 66, $2751-2754$ (2002)

21) Lee J. C., Lim K. T., Jang Y. S., Biochim. Biophys. Acta, 1570, 181191 (2002).

22) Peng Z. F., Strack D., Baumert A., Subramaniam R., Goh N. K., Chia T. F., Tan S. N., Chia L. S., Phytochemistry, 62, 219-228 (2003).

23) Dajcs J. J., Austin M. S., Sloop G. D., Moreau J. M., Hume E. B., Thompson H. W., McAleese F. M., Foster T. J., O’Callaghan R. J., Invest. Ophthalmol. Vis. Sci., 43, 1109-1115 (2002).

24) Lee K. Y., Shin D. S., Yoon J. M., Kang H., Oh K. B., J. Microbiol. Biotechnol., 12, 530-533 (2002).

25) British Society for Antimicrobial Chemotherapy, J. Antimicrob Chemother., 27(Suppl. D), 1-50 (1991).

26) Weiss W. J., Lenoy E., Murphy T., Tardio L., Burgio P., Projan S. J., Schneewind O., Alksne L., J. Antimicrob. Chemother., 53, 480-486 
(2004).

27) Jonsson I., Mazmanian S. K., Schneewind O., Bremell T., Tarkowski A., Microbes Infect., 5, 775-780 (2003).

28) Ton-That H., Liu G., Mazmanian S. K., Faull K. F., Schneewind O., Proc. Natl. Acad. Sci. U.S.A., 96, 12424-12429 (1999).

29) Grundmeier M., Hussain M., Becker P., Heilmann C., Peters G., Sinha B., Infect. Immun., 72, 7155-7163 (2004).

30) Hertog M. G. L., Hollman P. C. H., Katan M. B., J. Agric. Food Chem., 40, 2379-2383 (1992).
31) Hertog M. G. L., Hollman P. C. H., van de Putte B., J. Agric. Food Chem., 41, 1242-1246 (1993).

32) Cook N. C., Samman S., J. Nutr. Biochem., 7, 66-76 (1996).

33) Williamson G., Manach C., Am. J. Clin. Nutr., 81 (Suppl.), 243S255S (2005).

34) Cowan M. M., Clin. Microbiol. Rev., 12, 564-582 (1999).

35) Ashida H., Fukuda I., Yamashita T., Kanazawa K., FEBS Lett., 476, $213-217(2000)$ 